\title{
O sistema de informações estatísticas no Brasil e as relações entre seus produtores e usuários
}

\author{
Antonio Braz de Oliveira e Silva \\ Doutorando em ciência da informação pela Escola de Ciência da \\ Informação (ECI) da UFMG, especialista em economia pelo IEI. \\ (Instituto de Economia Industrial) \\ E-mail: antonio_braz@uol.com.br
}

\section{INTRODUÇÃO}

Os estudos sobre produção e uso de informação cobrem campo bastante amplo, com enfoques variados, e não estão livres de controvérsias. Essas pesquisas e outras ligadas à ciência da informação refletem as dificuldades inerentes ao fato de esta área ser uma área do conhecimento relativamente recente. Até hoje, quando se fala em ciência da informação, fala-se em uma área de conhecimento interdisciplinar, e não se delimita claramente o seu campo de atuação. Além disso, a discussão é sempre colocada em termos de sua relação com outras disciplinas (TARGINO, 1995; CARDOSO, 1996; GONZÁLEZ DE GÓMEZ, 2001; GOMES, 2003; LE COADIC, 1997).

A discussão sobre a natureza da ciência da informação parece ser tão antiga quanto a história desse campo de conhecimento. Foskett, por exemplo, já chamava a atenção para os efeitos pouco práticos da discussão e da "controvérsia entre bibliotecários e técnicos de informação" (FOSKETT, 1980, p. 14). De acordo com Vakkari (1991), durante os anos 70 essa discussão tinha um viés teórico e conceitual, ampliada no início dos anos 1990, incluindo a relação entre biblioteconomia e ciência da informação e, sobretudo, a identificação e definição dos conceitos centrais da disciplina, tais como conhecimento, informação e necessidade de informação.

Muitas pesquisas sobre a necessidade de informações poderiam ser agrupadas nas que destacam o papel da oferta - os sistemas de informação - e as que enfatizam o papel do usuário. As que deveriam examinar o encontro dos dois lados, dos usuários com os sistemas de informação, são desequilibradas para um dos dois lados mencionados (DERVIN; NILAN, 1986; FERREIRA, 1995; COSTA, 2000). Na verdade, a pesquisa centrada nos usuários adquire o status de paradigma, segundo a proposta de vários autores.

O paradigma que toma o usuário como o objeto primeiro da ciência da informação pode ser atribuído e associado, principalmente, aos estudos e publicações de Dervin (DERVIN; NILAN, 1986). Ele associa a necessidade de informação com o seu uso, e, por isso, também é descrito como paradigma da construção do sentido (sense-making paradigm).

\section{Keywords}

Information; Statistical information; Statistical system; Users of statistical information; Information society. 
Ainda que tenha aberto uma área de pesquisa de crescente importância na ciência da informação, não parece ter se consolidado como definidor de um novo e revolucionário programa de pesquisa*. Na verdade, parece caracterizar a necessidade de se realizarem estudos e utilizar conceitos e abordagens ad hoc, isto é, gerando resultados que não podem, ainda, ser generalizados e, conseqüentemente, que possam servir como base para avanços no campo. As pesquisas relacionadas aos processos cognitivos envolvendo os usuários e os sistemas de informação vêm crescendo de forma acentuada e com uma característica marcadamente multidisciplinar. Ainda assim:

Embora a teoria sobre a construção do sentido pareça atender aos critérios mínimos de um paradigma de pesquisa, não se pode, apressadamente, concluir que ocorrerá uma mudança revolucionária de paradigma [...]. A própria Dervin não exigia essa mudança, apesar das sérias anomalias que ameaçavam imobilizar a abordagem tradicional; ela pedia apenas uma atenção para a mudança de paradigma.

[...] não é realista entender que se trata de uma mudança radical na pesquisa em Ciência da Informação e Biblioteconomia e que toda a pesquisa passaria a ter como base a abordagem sense-making. (SAVOLAINEN, 1991, p. 160, original em inglês).

Analisando os desequilíbrios mencionados e as características dos seus problemas, Savolainen (1991) chama a atenção para o fato de o trabalho de Dervin fazer referências, também, aos aspectos estruturais (situacionais, sociais, econômicos e culturais). Esses aspectos estão presentes quando o indivíduo busca informação, como, por exemplo, as regras de acesso aos

\footnotetext{
* Um programa de pesquisa científica é um aglomerado de teorias conectadas que derivam de um núcleo comum, em outras palavras, o núcleo central composto pelas crenças comuns que unem os seguidores do programa. Para discutir a ciência da informação, González de Gómez (2000) utiliza esse conceito, introduzido por Imre Lakatos, historiador da ciência, na década de 60 , como ponto de partida. Esse conceito pode ser comparado ao de paradigma, desenvolvido por Thomas Kuhn (2003), e os seus conteúdos foram alvos de constantes debates entre seus autores, Lakatos e Kuhn, dois importantes filósofos da ciência e seguidores. Para a presente discussão, os dois conceitos se aproximam, uma vez que se está tratando das pesquisas que estruturam uma área de conhecimento. Para uma introdução ao trabalho de Lakatos, ver Larvor (1988). A aproximação dos dois conceitos para o tipo de discussão aqui apresentada já foi feita por outros autores, como na discussão sobre paradigmas tecnológicos feita por Dosi (1982): "One does not have any ambition here to argue "what science is" or tackle the epistemological disputes on the differences between the Kuhnian approach and Lakatlos 'one. For our purposes the degreee of overlap between the two approaches is great enough to borrow from them a few. basic dcfinitlons of science which they have in common".
}

sistemas de informação. Ainda segundo esse autor, essas regras não só limitam o comportamento individual, mas também facilitam sua busca, colocando à sua disposição recursos adequados. Assim, análises que levassem em conta os aspectos individuais e estruturais permitiriam melhor compreensão "dos interesses epistêmicos e práticos da disciplina” (SAVOLAINEN, 1991, p. 161). Dessa forma, apresenta-se como desafio à elaboração de pesquisas que tragam informações sobre esses dois aspectos de uma forma que facilitasse a sua análise. $\mathrm{Na}$ verdade, essa proposta agrega um componente social na necessidade de busca do indivíduo por informações.

Alguns sistemas de informação e seus usuários têm características que os tornam particulares para as análises acima mencionadas e deveriam ser tratados como um objeto composto pela interseção entre a oferta e a demanda de informações. Um exemplo é o sistema estatístico no qual produtores e usuários possuem as mesmas competências técnicas. As relações sociais, profissionais e institucionais são muito importantes para efetiva comunicação da informação, ou seja, para que ela aconteça, é necessária a existência de um "estoque considerável de conhecimento prévio comum" (FOSKETT, 1980, p. 31). Deve-se chamar a atenção que muitos usuários individuais são, na verdade, a face visível de uma ampla estrutura - as instituições de pesquisa. Esse sistema permaneceu em equilíbrio até que as recentes alterações no funcionamento das economias nacionais, causadas por mudanças significativas nas instituições em função de instrumentos legais vigentes até a década de 1980, e as amplificadas pelas novas tecnologias de informação e comunicação (TICs) romperam com esse estado.

Este artigo analisa o desenvolvimento do sistema estatístico e seu papel na oferta de informações, o caráter do seu relacionamento especial com o lado da demanda e as tranformações que as necessidades de um subconjunto específico de usuários vêm sofrendo em função da necessidade de acesso mais rápido a novas informações. Essas mudanças terão impacto nos serviços prestados pelos órgãos de estatística e na avaliação que a sociedade faz do seu funcionamento e padrão de qualidade .

\section{NOVOS TEMPOS}

Se fosse solicitado que as principais lideranças políticas e empresariais, personalidades do mundo das ciências, das artes, professores e pesquisadores descrevessem as principais transformações vividas a partir da segunda metade do século XX, certamente os elementos em comum estariam associados à produção crescente de informações e às tecnologias para seu processamento e 
disseminação. As etapas de produção, processamento e disseminação foram significativamente modificadas sobretudo em termos de eficiência e velocidade. As tecnologias de informação (e comunicação, ou simplesmente TICs) estão impondo uma "transformação na sociedade moderna em sociedade da informação, era da informação ou sociedade pós-industrial" (SARACEVIC, 1996, p. 42). Segundo o autor, essas tecnologias e as transformações por elas impostas à sociedade compõem uma das três características gerais que constituem a razão da existência da ciência da informação.

As mudanças decorrentes das TICs, apesar de se iniciarem no imediato pós-guerra, tiveram maior impacto nos últimos 30 anos e, por isso, estão associadas ao fenômeno recente denominado globalização*. Embora o termo em si não tenha uma definição precisa, há aceitação tácita, impressionista, que o associa às principais diretrizes do que ficou conhecido como Consenso de Washington: liberalização dos fluxos internacionais de capital e regimes de taxas de câmbio flutuantes, liberalização do comércio internacional, imposição aos Estados nacionais de políticas de austeridade fiscal, entre outras. De fato, as novas tecnologias fornecem a base para os movimentos econômicos mencionados. Assim, não se pode imaginar o volume e a velocidade dos fluxos de capital e sua reprodução e valorização em escala mundial, sem as tecnologias de comunicação e informação.

Embora impreciso, o termo globalização passou a identificar um mundo em constante mutação. Não por acaso, a área de administração de empresas e seus profissionais são os que mais utilizam o termo globalização para explicar as dificuldades na tomada de decisão, em especial nas áreas de marketing e planejamento. De acordo com Chesnais (1996), os principais consultores nessa área, com destaque para M. E. Porter, foram também os principais divulgadores do termo. A comunicação rápida dos fatos econômicos e políticos serviu para reforçar essa acepção do termo. No mundo dos negócios, isso significou não só a ampliação potencial dos mercados, mas também aumento do risco, tornando o ambiente de negócios ainda mais complexo e difícil de ser acompanhado. Para que as empresas e seus gerentes pudessem enfrentar os novos desafios, o uso de ferramentas adequadas, apoiadas sobre

\footnotetext{
* Alguns autores criticam o uso acadêmico do termo, como, por exemplo, Fiori (2001), no capítulo 6 "A palavra [globalização] foi cunhada na década de 90 , no campo da administração de empresas [...] Apesar da visível imprecisão conceitual, mesmo no campo acadêmico, transformou-se em palavra síntese que se propõe a designar, diagnosticar e explicar, ao mesmo tempo, todas as transformações vividas [...] nos últimos 25 anos do século XX".
}

informações precisas acerca da realidade, foram alçados à categoria de insumo fundamental e, portanto, estratégico para a sua sobrevivência. Assim, torna-se relevante, tanto do ponto de vista empresarial quanto acadêmico, "estudar as formas e meios pelos quais gerentes e outros profissionais obtêm informações a respeito do ambiente externo de suas organizações" (BARBOSA, 2002, p. 1).

Muito se discute sobre o acesso à informação, mas, de uma maneira geral, ele "tem sido dificultado, desde sempre, por razões econômicas" (DIAS, 2002, p. 4). Mas, por ser estratégica para as organizações em geral e as empresas em particular, deve-se buscar o desenvolvimento de estruturas que aperfeiçoem essas tarefas, ou seja, fazer com que essas atividades se desenvolvam com menor custo e maior eficiência. Como nenhuma organização ou empresa, mesmo de grande porte, teria condição de ser autárquica nessa área, ou seja, satisfazer apenas com recursos internos suas necessidades de informação, elas se valem de serviços de informação eficientes desenvolvidos e mantidos por outras organizações e empresas. Para que sejam eficazes, os serviços de informação devem se basear em diálogo genuíno com seus usuários, indo além da simples reação às perguntas apresentadas e da "manipulação sempre crescente de dados" (FOSKETT, 1980, p. 40).

Dentre as fontes de informação utilizadas, destacam-se aquelas produzidas pelas organizações públicas, especialmente os órgãos de estatística. Os serviços de informação estatística se destacam por buscar o fornecimento eficaz de informações e, de alguma forma, procuram implementar política de diálogo permanente com seus usuários. Na maior parte dos países do mundo, o órgão oficial de estatística responde pela produção de pesquisas econômicas, sociais e demográficas, além de coordenar a produção de informação realizada por outros órgãos, tais como o Banco Central, as agências de comércio exterior, os órgãos ligados ao mercado de trabalho etc. A sua atuação como produtor e coordenador está respaldada em recomendações de organismos internacionais, como o The United Nations Statistics Division (UNSD)* e o Fundo Monetário Internacional,

* As suas principais funções são: 1) coleta, processamento e disseminação das informações estatísticas (the collection, processing and dissemination of statistical information); 2) a padronização dos métodos estatísticos, classificações e definições (the standardization of statistical methods, classifications and definitions); 3) o programa técnico de cooperação com os países membros (the technical cooperation programme); 4) a coordenação das atividades e programas estatísticos internacionais (the coordination of international statistical programmes and activities). Ele é ligado ao Department of Economic and Social Affairs (http://www.un.org/esa/desa.htm) das Nações Unidas. 
recebendo, por isso, a denominação de coordenador do Sistema Estatístico Nacional (SEN). No Brasil, essa função é exercida pelo Instituto Brasileiro de Geografia e Estatística (IBGE), e os instrumentos legais que garantem a sua atuação* definem que todos os órgãos e entidades da administração pública direta e indireta de todas as esferas de governo e as entidades privadas que recebem subvenção ou auxílio para a produção de informações estatísticas fazem parte do Sistema Estatístico Nacional (SEN).

\section{A PRODUÇÃO DE ESTATÍSTICA E O SISTEMA ESTATÍSTICO NACIONAL}

A palavra estatística vem do alemão statistik e originalmente referia-se à ciência do Estado, ou seja, à descrição das coisas relevantes ao Estado e à tomada de decisão de seus governantes, tais como a sua população e o seu território (SENRA, 1999, p.2). Atualmente, referese a informações econômicas, sociais e demográficas obtidas por meio das pesquisas oficiais e dos registros administrativos** dos órgãos públicos e são, normalmente, definidas e aceitas como componentes do Sistema Estatístico Nacional***.

Embora a produção de estatísticas, especialmente demográficas, remonte a séculos, somente no século XVI foi associada claramente aos aspectos econômicos da sociedade, especialmente para fins tributários. Após a Revolução Industrial, no final do século XVIII, essa vertente se consolida, mas é no século XX, com a maior presença do papel do Estado na economia, que se constrói a noção de sistema estatístico. A situação enfrentada pela economia mundial ao final da década de 1930, com a recessão e o desemprego crescentes, trouxe imenso desafio para a ciência econômica: o de analisar e propor soluções para esse tipo de acontecimento. Surge então, como crítica à escola econômica dominante e como proposição de uma nova forma de ver a realidade econômica e formular políticas, o livro Teoria geral do emprego, do juro e do dinheiro, do economista inglês John

* O Decreto-Lei No 161 de 13/02/1967 tornou a instituição responsável pelo Plano Nacional de Estatística; a Lei 5878/73 instituiu o Plano Geral de Informações Estatísticas e Geográficas, que se tornou operacional com a Lei 6183 de 11/12/1974, que instituiu o Sistema Estatístico Nacional, sob a coordenação do IBGE.

** Os registros administrativos são informações mantidas pelos órgãos públicos na realização de sua tarefa principal, como, por exemplo, as informações sobre impostos que são mantidas pelas diferentes Secretarias de Receita. Se tratadas adequadamente, transformam-se em informações estatísticas de excelente qualidade e de baixo custo. *** Há quem prefira o termo Sistema de Informação Estatística, enfatizando a informação, isto é, o seu conceito, representação e contexto. Ver Porcaro (2001).
Maynard Keynes. Em linhas gerais, a Teoria Macroeconômica, a partir da Teoria Keynesiana, formulou um modelo de análise que conjuga os principais fatos econômicos: produção e geração da renda, consumo e poupança, acumulação e financiamento. A contrapartida foi o surgimento de um instrumento de mensuração que integra todas as informações existentes sobre as operações e agentes econômicos de uma forma conceitual e metodologicamente coerente denominado Sistema de Contas Nacionais, conforme apresentado a partir dos trabalhos de Richard Stone, também economista inglês, Prêmio Nobel de Economia de 1984*.

Dessa forma, a esquematização e quantificação das relações entre os agentes econômicos passaram a ser ordenadas por um sistema cuja referência era a Teoria Econômica. A produção de informações estatísticas passou a ter uma base científica.

Em contrapartida, as estatísticas sociais e demográficas careciam de um referencial semelhante, o que levou as Nações Unidas a propor um sistema que as integrasse entre si e com as estatísticas econômicas. À frente dos trabalhos, o mesmo Richard Stone, tendo como resultado a publicação do relatório técnico denominado Towards a System of Social and Demographic Statístics (UNITED NATIONS, 1975).

Na introdução, o autor discute, ainda que de forma rápida, alguns aspectos relacionados ao sistema (o que é um sistema, seu escopo e tipos de informação que estarão contempladas, suas conexões e como construí-lo) e aos seus usos e usuários. Resumidamente, a idéia de sistema está intimamente ligada à idéia de conexão entre suas partes. A realidade social é por demais complexa, de forma que as estatísticas são coletadas e apresentadas fragmentadas (por exemplo, informações sobre saúde, educação, trabalho) e os diferentes campos de conhecimento envolvidos não conseguiram ainda criar, em virtude da complexidade da tarefa, as conexões necessárias ao sistema. No entanto, para superar essas dificuldades e caminhar na direção de construção do sistema, devem-se fazer hipóteses sobre como o mundo real funciona. Resta saber como definir as hipóteses, dada a diversidade de abordagens e escolas em cada área do conhecimento. Assim:

\footnotetext{
* "for having made fundamental contributions to the development of systems of national accounts and hence greatly improved the basis for empirical economic analysis", conforme The Bank of Sweden Prize in Economic Sciences in Memory of Alfred Nobel. Disponível em <http:/ /www.nobel.se/economics/laureates/1984/index.html>.
} 
Uma vez que os dados no sistema devem ser utilizados para grande variedade de propósitos analíticos, assim como para a sua descrição precisa, é necessário que eles sejam de determinada natureza e dispostos em organização tal que sejam capazes de atender às necessidades dos principais tipos de modelos que são utilizados nos diferentes campos de interesse (UNITED NATIONS, 1975, p. 3, original em inglês.).

O objetivo do sistema estatístico é prover a sociedade de informações para o conhecimento e transformação da realidade social. Deve possibilitar, ainda, que as preocupações de produzir informações amplas e integradas entre si, conforme mencionado em várias publicações das Nações Unidas, sejam, ao menos em parte, atendidas. As recomendações internacionais sobre a produção de informações estatísticas são genéricas, cabendo a cada um dos Estados nacionais definir suas prioridades. Países em diferentes estágios de desenvolvimento econômico e social devem definir as prioridades na implantação do seu sistema estatístico, levando em conta a sua realidade e suas limitações orçamentárias. Nessa linha, o IBGE buscou ouvir os principais usuários de suas informações. No entanto, o fez de forma nãosistemática e regular. Ainda assim é reconhecido, tanto pela imprensa quanto pela academia, como um órgão que reúne profissionais competentes realizando tarefas relevantes para o conhecimento da realidade nacional. Se não existe forma regular de consulta, como saber se a sociedade (ou pelo menos a parcela representada pelos pesquisadores e demais usuários de informações estatísticas) está atendida em sua demanda por informações? Ou, em outras palavras, se as consultas são irregulares, como explicar a aprovação do que é produzido, ou seja, a qualidade das informações estatísticas?

\section{SISTEMA ESTATÍSTICO COMO SISTEMA ESPECIALISTA}

A avaliação de sistemas de informação apresenta dificuldades tanto do ponto de vista teórico quanto prático. Em primeiro lugar, o próprio conceito de qualidade, uma vez que as definições usadas são, normalmente, bastante superficiais (PAIM, NEHMY, 1996, p.81). Diversos modelos teóricos para a avaliação de sistemas de informação apontam atributos a avaliar, mas não definem claramente o modo como devem ser avaliados (AROUCK, 2001, p.7). Mesmo considerandose os diferentes aspectos da qualidade da informação e a obrigação de analisá-la de forma multidimensional, do ponto de vista de sistemas de informação, há, muitas vezes, confusão entre o conteúdo e a forma de prestação dos serviços.
$\mathrm{Na}$ tentativa de se evitar o excesso de subjetivismo e adotando-se as dimensões intrínseca e contingencial para se analisar a qualidade da informação*, pode-se afirmar, em primeiro momento, que a primeira (que engloba os atributos validade, confiabilidade, precisão, completeza, novidade, atualidade, significado através do tempo e abrangência) está associada aos sistemas e serviços de informação, enquanto a segunda (que engloba os atributos valor percebido, eficácia, relevância e redundância, além de características relativas ao formato do produto) está associada ao usuário. Para trazer para os termos mais usados na área de estatísticas, a primeira está relacionada à oferta, enquanto a segunda está relacionada à demanda por informações.

Embora as diferenças entre as dimensões tendam a desaparecer na prática da avaliação da informação, devese buscar entender como esse processo acontece no campo das informações estatísticas. A oferta de informações estatísticas é, muitas vezes, analisada do ponto de vista técnico-científico, ou seja, a definição do que pesquisar está intimamente associada a um corpo teórico multidisciplinar que, conforme já mencionado, é a base do sistema estatístico, embora algumas áreas ainda não se organizem integralmente como sistema. Dessa forma, os membros do corpo técnico envolvidos com as atividades de produção de informações são selecionados de acordo com sua formação nas áreas de ciências humanas aplicadas (economia, sociologia etc.) e nos métodos quantitativos que lhes dão suporte (estatística, matemática, ciência da computação etc.). Essa característica permite, também, a participação desses técnicos nos fóruns internacionais de debate e traz para a instituição a aceitação entre os seus pares de outros países. São essas características que conferem reputação ao órgão oficial de estatística e, por conseqüência, ao sistema estatístico que ele coordena, permitindo que o IBGE mantenha posição de destaque na América Latina (GRACIOSO, 2003). Ou ainda, a condição de sistema especialista ou perito.

Dentre os usuários, é possível definir dois grupos a partir da forma dominante de acesso às informações: o dos usuários que trabalham em grandes instituições de pesquisa públicas ou privadas que têm necessidade de acesso rápido e utilizam grande volumes de dados como base para a sua produção científica e empresarial e o daqueles que utilizam informações estatísticas de forma

* São definidos três dimensões: transcendental, intrínseca e contingencial, mas mencionam que muitos estudiosos consideram a dimensão transcendental como equivalente à intrínseca (PAIM; NEHMY, 1996, 1998). Dessa forma, aqui foram consideradas apenas duas dimensões. 
eventual para a solução de problemas bem definidos. Em termos de acesso, os primeiros preferem o acesso direto aos bancos de dados ou aos produtos em meio magnético, e os outros preferem as publicações, mesmo depois que sua busca inicial seja complementada por acesso aos meios eletrônicos (CD ou Internet). Os primeiros mantêm, de forma dominante, contatos institucionais e impessoais, enquanto aqueles do segundo grupo normalmente mantêm contatos pessoais (em consultas à biblioteca, pessoalmente ou por telefone).

A existência do primeiro grupo, que na verdade é constituído pelos maiores usuários de informação e formadores de opinião, faz com que as dimensões intrínseca e contingencial sejam, na prática, pouco relevantes para a análise do sistema de informação estatística. Os produtores e usuários compartilham da mesma formação técnico-científica, participam dos mesmos fóruns de discussão e das mesmas associações profissionais, ou seja, compartilham o mesmo modelo mental com relação à produção de estatísticas*. As eventuais divergências estão associadas às prioridades, que decorrem, em grande medida, de restrições orçamentárias.

Já o segundo grupo de usuários, embora possa ter essas mesmas características, tende a fazer sua avaliação das estatísticas de outra forma. Pesquisas realizadas pelo IBGE ao longo dos últimos dois anos indicam que critérios relativos à qualidade do serviço prevalecem. No caso do atendimento pessoal, as relações estabelecidas com os funcionários, a presteza no atendimento e as instalações existentes são elementos fundamentais nessa avaliação.

De acordo com essa análise, a situação do sistema de informações estatísticas estaria estabilizada do ponto de vista da relação entre oferta e demanda, ou entre produtores e usuários de informação. Novas pesquisas seriam realizadas quando os recursos estivessem disponíveis, com a qualidade já reconhecida de informação já existente. As novas tecnologias de

\footnotetext{
* Buscando, de forma um pouco livre e ousada, apoio nos conceitos apresentados por Bourdieu (2000), pode-se dizer que, no campo da produção de informações estatísticas, os produtores, ligados às grandes instituições públicas tais como o IBGE, o Banco Central e os Ministérios, e os principais usuários, ligados a outras instituições públicas, como o Instituto de Pesquisa Econômica Aplicada (Ipea) e as universidades, e às grandes empresas compartilham os mesmas posições dominantes e possuem capital intelectual equivalente, ou seja, competências equivalentes. Ainda segundo esse autor, o campo é definido como o locus das lutas no qual os agentes buscam manter ou alterar a distribuição do capital (específicos ao campo, isto é, relevantes), assim como a sua posição dentro dele. Essa situação de equilíbrio vai se alterar com as novas demandas por parte de uma parcela dos usuários, lembrando que eles ocupam posições dominantes no campo.
}

informação e comunicação trouxeram importantes transformações no custo de reproduzir informação e ampliaram o acesso a ela. Nas palavras dos usuários representados no Fórum Nacional de Usuários, informar é um ato político, e as informações sociais, econômicas e territoriais devem contribuir para o aperfeiçoamento do processo democrático e de tomada de decisões (IBGE, 1996). No entanto, essas mesmas tecnologias trouxeram novos desafios, tanto na forma de produção quanto divulgação das pesquisas. A ampliação do número de usuários, a velocidade percebida nas formas de comunicação e as possibilidades de cruzamento de dados permitidas pelos computadores pessoais impõem novos desafios ao órgão produtor de informações estatísticas.

\section{O SISTEMA ESTATÍSTICO E A SOCIEDADE DA INFORMAÇÃO: NOVOS DESAFIOS}

As transformações recentes no ambiente tecnológico internacional significaram "uma mudança no paradigma das tecnologias intensivas em capital e energia e de produção inflexível e de massa (baseadas em energia e materiais baratos) para as tecnologias intensivas em informação, flexíveis e computadorizadas" (CASSIOLATO, 1999, p.164). As tecnologias de informação e comunicação (TICs) são a base para o que se denominou, também de forma imprecisa, sociedade da informação. Essa denominação é apenas uma dentre as que surgiram nas últimas duas ou três décadas do século XX, tais como economia da informação, economia do aprendizado, economia do conhecimento ou economia da inovação. Seu aspecto mais importante é que o conhecimento é o seu recurso mais estratégico e que o acesso à informação para a geração de novos conhecimentos é fundamental nesse processo.

O sistema estatístico foi definido tendo como paradigma a sociedade industrial e a organização da produção dela decorrente. O novo paradigma está trazendo novos desafios com relação à produção de informações, uma vez que altera significativamente a organização das atividades produtivas (em muitos casos, organizada em redes, que podem, inclusive, ultrapassar as fronteiras nacionais), as novas formas de organização do trabalho (as subcontratações mais tradicionais têm sido muito modificadas, ampliando-se as possibilidades do trabalho à distância realizados em novas bases, com profissionais de alta qualificação e com uso intensivo das TICs), a valoração dos ativos intangíveis etc.

Em contrapartida, os principais usuários de informações estatísticas, principalmente os que trabalham para as grandes empresas do setor privado, exigem maior rapidez 
na divulgação de informações, sistemas mais amigáveis de recuperação e o uso de novas tecnologias, como, por exemplo, as informações georreferenciadas. Entre os dois grupos de usuários mencionados, está havendo diferenciação no primeiro corroborando para o surgimento de um terceiro, formado por empresas de grande porte que necessitam de novas informações e de maior rapidez no acesso para suas atividades de inteligência competitiva, cada vez mais complexas e estratégicas e, portanto, não delegáveis a outras empresas. Esse grupo não tem sido atendido na velocidade imposta pelos novos tempos.

\section{CONSIDERAÇÕES FINAIS}

As novas demandas da sociedade impõem novos desafios para os serviços de informação, em especial aqueles relacionados com a produção de estatísticas. Até recentemente, o relacionamento entre os produtores e os grandes usuários dessas informações tinha certa estabilidade em função do forte relacionamento profissional entre eles. Os usuários eventuais de informações estatísticas eram parcialmente atendidos e, ainda que sentindo falta de mais informações, não possuíam capacidade de articulação para formular críticas ou novas demandas ao Sistema Estatístico Nacional. As mudanças causadas pela introdução de novas tecnologias de informação e comunicação (TICs) e a demanda por maior rapidez e maior abrangência das informações, especialmente para atender ao setor produtivo, ávido por informações sobre os seus ambientes de atuação, são os fatos notórios observados ao longo dos anos 1990.

Ante esse quadro, o sistema estatístico nacional e o seu coordenador, o órgão oficial de estatística do país (IBGE), estão diante de um novo desafio que transcende as discussões habituais sobre a qualidade das informações estatísticas. Apenas como referência inicial, dados que ampliam a discrepância entre as estatísticas oferecidas pelo sistema atual e as novas demandas, sugere-se que se faça uso de sistemas como o Gap Model. Devem-se identificar os atributos nos quais as diferenças entre o que é desejado e o que é percebido pelos usuários são significativas, como forma de identificar deficiências no sistema estatístico. Deve-se mencionar que a oferta de novas estatísticas será sempre restringida pelos aspectos orçamentários, já que envolvem recursos expressivos, principalmente quando se considera a dimensão do Brasil. No entanto, novos produtos, novos cruzamentos de dados e aplicativos mais amigáveis podem ser desenvolvidos para atender a novas demandas. De qualquer forma, a relação entre produtores e usuários deverá se adaptar aos novos rumos da economia e da sociedade. Para a ciência da informação, essa área pode constituir laboratório para a pesquisa aprofundada sobre o comportamento do indivíduo ante uma estrutura, aqui definida como o sistema de informações estatísticas. Essa estrutura constitui a referência para o comportamento de um conjunto de usuários com características especiais e os produtores de informação estatística, agregando-se, assim, um componente social na necessidade de busca do indivíduo por informações, componente esse presente, em grande medida, nos produtores e gestores do sistema estatístico.

\section{AGRADECIMENTOS}

autor realizou o presente trabalho sob o apoio do Programa de Pós-Graduação IBGE. Meus agradecimentos aos colegas Wladmir Brandão, Marcus José Campos e Renato Matheus e aos avaliadores anônimos pelas sugestões que permitiram que este artigo fosse substancialmente melhorado. As falhas remanescentes são de minha inteira responsabilidade.

Artigo recebido em 31/01/2005 e aceito para publicação em 05/01/2006. 


\section{O sistema de informações estatísticas no Brasil e as relações entre seus produtores e usuários}

\section{REFERENCIAS}

AROUCK, Osmar. Avaliação de sistemas de informação: revisão da literatura. Transinformação, v. 13. n. 1, p. 7-21, jan./jun. 2001.

BARBOSA, Ricardo Rodrigues. Inteligência empresarial: uma avaliação de fontes de informação sobre o ambiente organizacional externo. DataGramaZero: revista de ciência da informação, v. 3, n. 6 , dez. 2002. Disponível em: <http://www.dgz.org.br/dez02/ F_I_aut.htm $>$. Acesso em: 10 out. 2003.

BOURDIEU, Pierre. O campo econômico. Campinas: Editora Papirus, 2000 .

CASSIOLATO, José Eduardo. A economia do conhecimento e as novas políticas industriais e tecnologias. In: LASTRES, Helena; ALBAGLI, Sarita Albagli (Org.). Informação e globalização na era do conhecimento. Rio de Janeiro: Ed. Campus, 1999.

CARDOSO, Ana Maria Pereira. Pós modernidade e informação: conceitos complementares?. Perspectivas em Ciência da Informação, Belo Horizonte, v. 1, n. 1, p. 63-79, jan./jun. 1996.

CHESNAIS, Fraçois. A mundialização do capital. 1. ed. São Paulo: Xamã Editora, 1996

COSTA, Cândida Aleixo de Oliveira. Aplicação da abordagem sensemaking no estudo do comportamento informacional de pesquisadores de um instituto de pesquisa tecnológica. 2000. 94 f. Dissertação (Mestrado)Ciência da Informação, Departamento de Ciência da Informação, UFMG, Belo Horizonte, 2000.

DERVIN, B.; NILAN, M. Information needs and uses. Annual Review of Information Science and Technology, v. 21, p. 3-33, 1986.

DIAS, Eduardo Wense. Ensino e pesquisa em ciência da informação. DataGramaZero: revista de ciência da informação, v. 3, n. 5, out. 2002. Disponível em: <http://www.dgz.org.br/out02/F I aut.htm> Acesso em: 10 out. 2003.

DOSI, Giovanni. Technological paradigms and technological trajectories: a suggested interpretation of the determinants and directions of technical change. Research Policy, n. 11, p. 147-162, 1982.

ENCONTRO NACIONAL DE PRODUTORES E USUÁRIOS DE INFORMAÇÕES SOCIAIS, ECONÔMICAS E TERRITORIAIS; FÓRUM NACIONAL DE USUÁRIOS, 3, 1996, Rio de Janeiro. Anais... Rio de Janeiro: IBGE, 1996. Sessões 10-14. Mimeografado.

FERREIRA, Sueli Mara Pinto. Novos paradigmas e novos usuários da informação. Ciência da Informação, Brasília, v. 25, n. 2, 1995. Disponível em: <http://www.ibict.br/cienciadainformacao/viewissue.php?id=31>. Acesso em: 10 out. 2003.

FIORI, José Luis. 60 lições dos 90: uma década de neoliberalismo. 1. ed. Rio de Janeiro: Editora Record, 2001.

FOSKETT, D. J. Informática. In: GOMES, Hagar Espanha. (Org.). Ciência da informação ou informática?. Rio de Janeiro: Calunga, 1980. p. 1-51. (Ciência da Informação).

GOMES, Henriette Ferreira. Interdisciplinaridade e ciência da informação: de característica a critério delineador de seu núcleo principal. DataGramaZero: revista de ciência da informação, v. 2, n. 4, ago. 2001. Disponível em: < http://www.dgz.org.br/>. Acesso em: 10 out. 2003.
GONZÁLEZ GÓMEZ, Maria Nélida. Para uma reflexão epistemológica acerca da ciência da informação. Perspectivas em Ciência da Informação, Belo Horizonte, v. 6, n. 1, p. 5-18, jan./jun. 2001.

Metodologia de pesquisa no campo da ciência da informação. DataGramaZero: revista de ciência da informação, v. 1, n. 6, dez. 2000. Disponível em: <http://www.dgz.org.br/dez00/ F I aut.htm>. Acesso em: 10 out. 2003.

GRACIOSO, Luciana de Souza. Disseminação de informações estatísticas no Brasil: práticas e políticas das agências estaduais de estatística. Ciência da Informação, Brasília, v. 32, p. 69-76, maio/ago. 2003. Disponível em: < http://www.ibict.br/cienciadainformacao/ include/getdoc.php $? \mathrm{id}=436 \&$ article $=152 \&$ mode $=\mathrm{pdf}>$. Acesso em: 10 out. 2004

KUHN, Thomas S. A estrutura das revoluções científicas. 7. ed. São Paulo: Editora Perspectiva, 2003. 262 p.

LE COADIC, Yves-François. A ciência da informação. Brasília: Briquet de Lemos/Livros, 1996. 119 p. ISBN 8585637080.

LARVOR, Brendan. Lakatos, an introduction. London: Routledge, 1998.

NAÇÕES UNIDAS. Towards a system of social and demographic statistics. New York: United Nations, 1975. (Studies in Methods, serie F, n. 18).

PAIM, Isis; NEHMY, Rosa Maria. A desconstrução do conceito de "qualidade da informação". Ciência da Informação, Brasília, v. 27, n. 1, p. 36-45, jan./abr. 1998.

- Questões sobre a avaliação da informação: uma abordagem inspirada em Giddens. Perspectiva em Ciência da Informação, Belo Horizonte, v. 3, n. 1, p. 81-95, jul./dez. 1996.

PORCARO, Rosa Maria. A informação estatística oficial na sociedade da informação: uma (des)construção. DataGramaZero: revista de ciência da informação, v. 2, n. 2, abr. 2001. Disponível em: < http:// www.dgz.org.br/abr01/F_I_aut.htm>. Acesso em: 10 out. 2003.

SARACEVIC, T. Ciência da informação: origem, evolução e relações. Perspectiva da Ciência da Informação, Belo Horizonte, v. 1, n. 1, p. 41-62, jan./jun. 1996.

SAVOLAINEN, Reijo. The sense-making theory - an alternative to intermediary-centered aproaches in library and information science? In: VAKKARI, Pertti; CRONIN, Blaise (Ed.). CONCEPTIONS OF LIBRARY AND INFORMATION SCIENCE - HISTORICAL, EMPIRICAL AND THEORETICAL PERSPECTIVES INTERNATIONAL CONFERENCE, 1991, Finland. Proceedings... London: Taylor Graham, 1991.

SENRA, Nelson de Castro. Informação estatística: política, regulação, coordenação. Ciência da Informação, Brasília, v. 28, n. 2, 1999. Disponível em: < http://www.ibict.br/cienciadainformacao/viewissue.php?id=22>. Acesso em: 10 out. 2003.

TARGINO, Maria das Graças. A interdisciplinaridade da ciência da informação como área de pesquisa. Revista Informação $\mathscr{E}$ Sociedade, v. 5, n. 1, 1995. Disponível em: < http://www.informacaoesociedade.ufpb. br/519501.pdf>. Acesso em: 28 jun. 2004.

VAKKARI, Pertti L. Open the horizon of expectations. In: VAKKARI, Pertti; CRONIN, Blaise (Ed.). CONCEPTIONS OF LIBRARY AND INFORMATION SCIENCE - HISTORICAL, EMPIRICAL AND THEORETICAL PERSPECTIVES INTERNATIONAL CONFE. RENCE, 1991, Finland. Proceedings... London: Taylor Graham, 1991. 